\title{
ESTUDIO DE PLANTAS MEDICINALES EN LA AMAZONÍA PERUANA: UNA EVALUACIÓN DE OCHO MÉTODOS ETNOBOTÁNICOS
}

\section{Lars Peter Kvist*, Isabel Oré $^{* *}$, Andrea Gonzales ${ }^{* *}$, Consuelo Llapapasca ${ }^{* *}$.}

\section{RESUMEN}

Desde 1993 hasta 1998, se realizaron estudios de plantas medicinales en comunidades de la Reserva Nacional Pacaya-Samiria. Se aplicó ocho métodos para identificar especies que sirven a las poblaciones locales como medicinas con la finalidad de describir cómo estas las manejan y usan. En este estudio, se caracterizan y clasifican los ocho métodos usados investigando las fortalezas y debilidades de cada uno de estos. Asimismo, se identifican y discuten 11 objetivos principales para estudios de plantas medicinales y nueve parámetros adicionales útiles para medir ventajas y desventajas de los métodos. Se concluye que cada método contribuye de manera significativa a varios, pero nunca a todos los objetivos y que siempre hay factores que favorecen o dificultan los métodos, debido tanto a los recursos y medios disponibles para el estudio como al entorno en que este se realiza. Así, la selección de métodos para el estudio de plantas medicinales deberá tener como base una cuidadosa precisión de objetivos y un análisis de las condiciones de la zona. En muchos casos, es necesario combinar varios métodos para cumplir con los objetivos definidos. Para ello, se aplican los métodos seleccionados en un orden lógico.

Palabras clave: Plantas medicinales, métodos, etnobotánica, ventajas, desventajas.

\begin{abstract}
From 1993 up to 1998 medicinal plants were studied in communities of the National Reservation Pacaya-Samiria, applying eight methods to identify species used by the local populations for medicine and to investigate how they manage and use the plants. The eight methods are characterized and classified and their respective strengths and

* Agencia Danesa para el Desarrollo (DANIDA). Copenhague (Dinamarca). Correo electrónico: lars_peter_kvist@hotmail.com

** Universidad Nacional de la Amazonía Peruana (UNAP). Iquitos (Perú). Correos electrónicos: isabeo@hotmail.com; consuelo_kvist@hotmail.com
\end{abstract}


weaknesses are discussed. To that end are defined 11 objectives for studies of medicinal plants as well as nine additional parameters useful to measure advantages and disadvantages of the particular methods. The conclusions are that each method contributes significantly to several but never to all objectives, and that combinations of additional parameters favor and disfavor any method, related to the resources and means present, preconditions for the study and conditions in the study area. This imply, that the selection of methods for any study of medicinal plants, should be based on a careful definition of its objectives and an analysis of the environment of the study. In most cases the best option is to combine several methods, applying the selected methods in a logical order.

Key words: Medicinal plants, methods, ethnobotany, advantages, disadvantages.

\section{INTRODUCCIÓN}

Muchos investigadores han publicado estudios etno-botánicos a partir de información provista por una o pocas personas. Considerando que los conocimientos de las personas de las comunidades locales varían mucho (Alcorn, 1981; Phillips y Gentry, 1993b) y que los informantes probablemente siempre proveen algunas informaciones incorrectas (Bernard et al., 1984; Godoy y Lubowski, 1992), se han criticado estudios etno-botánicos por tener resultados casuales y no permitir pruebas de hipótesis estadísticas, además por la falta de una minuciosa descripción de los métodos (Johns et al., 1990; Phillips \& Gentry, 1993a; 1993b). Partiendo de estas observaciones, Kvist (1997) describió y comparó cuatro métodos que permitían una descripción cuantitativa de patrones de uso de plantas.

El presente trabajo muestra una descripción, clasificación, evaluación y comparación de ocho métodos etno-botánicos y etno-farmacológicos, que fueron comprobados dentro del contexto de tres proyectos financiados por la Agencia Danesa para el Desarrollo (DANIDA) y realizados en colaboración con tres instituciones ubicadas en Iquitos: el IIAP (Instituto de Investigaciones de la Amazonía Peruana), el Herbario de la UNAP (Universidad Nacional de la Amazonía Peruana) y el Proyecto Integral de Desarrollo y Conservación Pacaya-Samiria (WWF/AIF/DK). Uno de estos proyectos consistió en el estudio específico de plantas con propiedades antiparasitarias; los otros dos proyectos estaban referidos al estudio de plantas medicinales, pero fueron más amplios, puesto que involucraron aspectos de la diversidad, el potencial económico y el manejo de los bosques inundables. 
El objetivo de este trabajo es el de facilitar que las personas interesadas en el estudio de las plantas medicinales seleccionen los métodos adecuados para los objetivos definidos en sus trabajos. Aquí se demuestra que cualquier método representa una combinación de ventajas y desventajas y que cualquier estudio debe definir sus métodos a partir de sus objetivos, de sus recursos y de su entorno.

\section{METODOLOGÍA}

\section{1. Área de estudio}

El área de estudio corresponde a la Reserva Nacional Pacaya-Samiria (RNPS), ubicada en la selva baja de la Amazonía Peruana, departamento de Loreto que, con 2080000 ha, se constituye en una de las áreas protegidas más grandes del Perú. Los estudios efectuados se desarrollaron en la periferia de la RNPS en la denominada zona de influencia, en comunidades asentadas en los ríos Marañón, Ucayali y el canal del Puinahua (Figura 1). La población local habla español y la mayoría es descendiente de Cocama-Cocamillas. Algunos preservan su identidad indígena y se organizan como comunidades nativas. Tal es el caso de las comunidades ubicadas en el canal de Puinahua y en Samiria (río Marañón). Casi todos los estudios discutidos se realizaron en bosques inundables y algunos en parcelas permanentes cerca de Jenaro Herrera. Kvist y Nebel (2000) ofrecen una introducción general a los bosques inundables de la zona y Nebel et al., (2000a; 2000b) describen las parcelas permanentes.

\subsection{Estrategias de evaluación}

- Describir los métodos usados para caracterizar cada uno de ellos evaluándolos y comparándolos entre sí.

- Identificar objetivos básicos y parámetros adicionales necesarios en estudios de plantas medicinales.

- Valorar los métodos confrontando cada uno de ellos con los objetivos básicos y parámetros identificados; evaluar los métodos de acuerdo con las categorías establecidas. El resultado será interpretado a partir de una escala de valores propuesta.

- Determinar individualmente las ventajas y desventajas de los métodos. Las conclusiones y recomendaciones presentadas parten de criterios y experiencias propias. 
Figura 1. Área del Estudio.

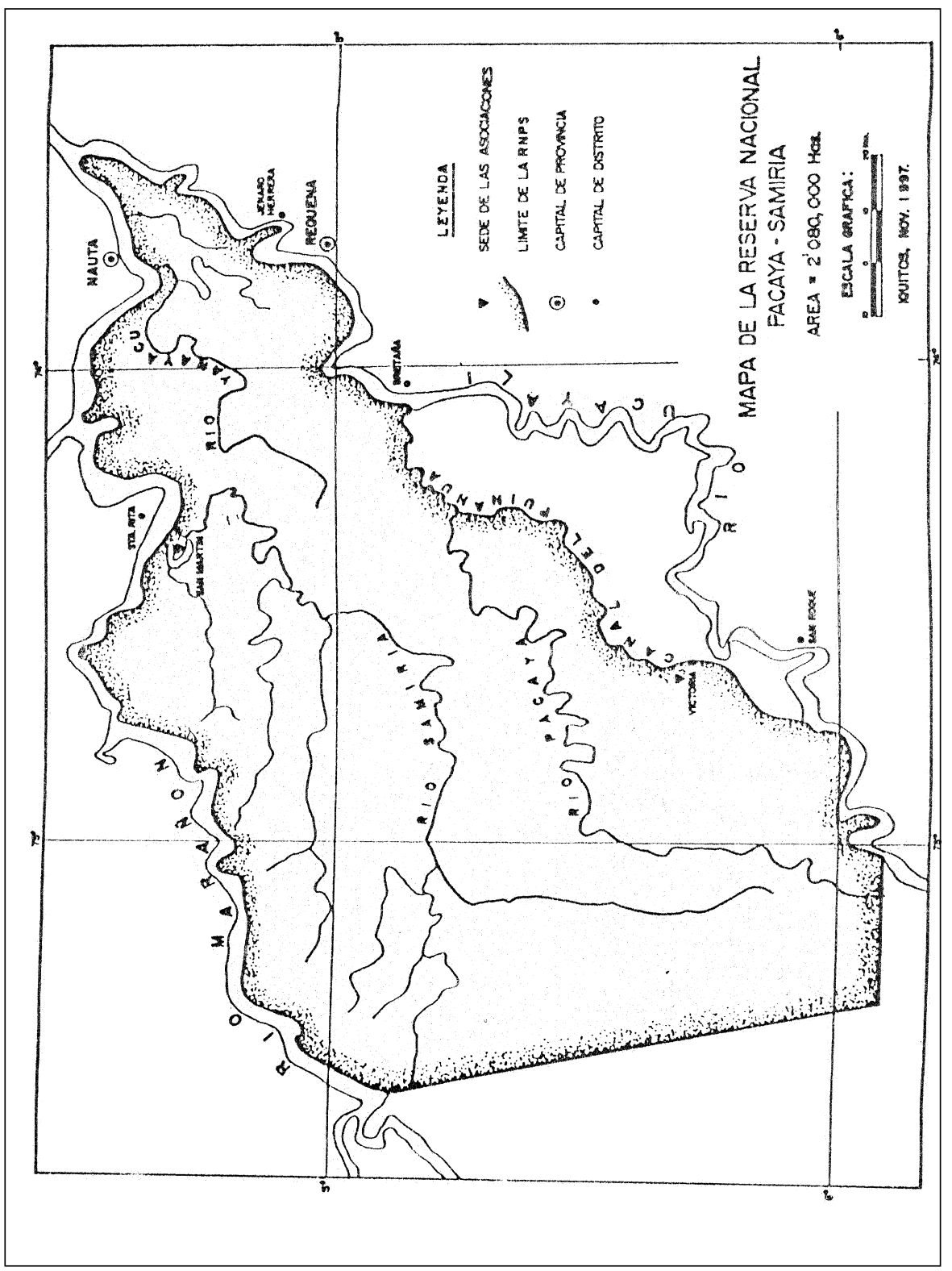




\subsubsection{Descripción de los métodos evaluados}

Gram (2001), Gram et al., (2001), Gonzales et al., (1999, 2000), Kvist (1997), Kvist et al., (1995, 1998, 2001a, 2001b), Oré et al., (1999) y Stagegaard et al., (2001) presentan, en detalle, los métodos evaluados en el presente estudio y los resultados encontrados. Aquí se presenta una descripción sistematizada de los ocho métodos aplicados:

\section{A. Estudio etno-botánico cualitativo}

En 1993, trabajando con un informante principal, se realizó un estudio preliminar de las plantas medicinales conocidas en la zona de Jenaro Herrera. El estudio obtuvo como resultado una lista de 120 plantas medicinales y 210 usos registrados (Kvist et al., 1998). Este método no realiza evaluaciones del valor o importancia relativa de las diferentes plantas medicinales y tampoco facilita análisis estadísticos de resultados. Por eso, a diferencia de los demás trabajos, que son considerados cuantitativos, se considera este trabajo como cualitativo.

\section{B. Usos potenciales de plantas medicinales elegidas por investigadores}

En 1994, se llevaron informantes de comunidades locales a las parcelas permanentes, cerca de Jenaro Herrera. A través de una entrevista, se les preguntó sobre los usos de árboles y bejucos elegidos por los investigadores. Cada planta (417 árboles y lianas) fue presentada independientemente a un mínimo de seis diferentes informantes. Se realizaron así entrevistas estructuradas y estandarizadas (Kvist et al., 1995; 2001a; Stagegaard et al., 2001). La característica principal de este método consiste en que las plantas en cuestión fueron elegidas de antemano por los investigadores $y$, posteriormente, presentadas a los informantes.

Además, en lugar de registrar la extracción y los usos de las plantas realizados por los informantes (métodos $\mathrm{G}, \mathrm{H}$ ), se registraron los usos potenciales de las plantas, es decir, los informantes indicaron para qué fines pueden servir estas (al igual que en los métodos A, C, D, E, y F).

\section{Usos potenciales de plantas medicinales elegidas por informantes}

En 1995, se trabajó con 13 informantes, 7 hombres y 6 mujeres, en diferentes comunidades en la zona de Jenaro Herrera. Los informantes incluidos eran conocedores de plantas medicinales (parteras y curanderos). Se trabajó dos días con cada informante. En este lapso, buscando y recolectando las plantas, se recopiló información sobre las plantas medicinales apreciadas por el informante. Sin embargo, se aceptó informa- 
ción sobre plantas que no se encontraron. Cada uno de los 13 informantes mencionó entre 50 y 80 plantas medicinales. Para cada planta, se realizó una entrevista estructurada y estandarizada. Aproximadamente, 170 taxas medicinales fueron reportadas y discutidas (Kvist, 1997; Kvist et al., 1998). La característica principal consiste en que los investigadores dejaron que los informantes eligieran las plantas mientras realizaban las entrevistas sobre sus usos potenciales.

\section{Plantas útiles para enfermedades específicas}

Desde 1995 hasta 1997, se entrevistaron familias de diferentes comunidades sobre sus conocimientos y/o usos de plantas para las enfermedades de malaria y leishmaniasis. En cada caso, se realizaba una entrevista estructurada y estandarizada. Además, se buscaba las plantas correspondientes a las enfermedades (Gonzales et al., 1999, 2000). Este método (tanto como el E) está relacionado con el anterior (método C). Los informantes buscaron las plantas y reportaron sus usos potenciales, pero bajo criterios definidos por los investigadores. Además, se entrevistaron familias (en sus hogares) en lugar de informantes aislados.

\section{E. Plantas medicinales conocidas bajo nombres vernaculares}

Entre 1997 y 1998, se buscaron ciertas plantas a partir de sus nombres vernaculares con el propósito de enfocar el estudio en especies medicinales reconocidas por la población y averiguar la consistencia de los nombres vernaculares reportados. Estos trabajos se realizaron en Jenaro Herrera, Puinahua y Samiria. Los informantes identificaron las plantas que conocían bajo nombres locales definidos. Se recolectaron muestras botánicas, cuya información se registró de manera específica con respecto a sus usos. El criterio diferente del investigador y el hecho de trabajar con informantes aislados distingue este método del anterior.

\section{F. Usos medicinales potenciales registrados mediante inventarios participativos}

Entre 1997 y 1998, junto con informantes locales, se realizaron inventarios en bosques, chacras y huertos de las zonas Samiria y Puinahua para identificar plantas a partir de nombres vernaculares y registrar sus usos potenciales. En los bosques, se hizo un inventario de los árboles ubicados dentro de fajas correspondientes a 1 ha: 10 metros de ancho y 1000 metros de largo. En el caso de chacras y huertas caseras, se trabajó con sus dueños registrando cultivos, otras plantas y apuntando sus usos (Oré et al., 1999). Con este método, ni el investigador ni el informante eligen las plantas específicas por discutir y evaluar. Sin embargo, el investigador define dónde hacer el inventario, de manera que este influye y restringe la selección de plantas y hábitats estudiados. 


\section{G. Recopilación de uso de plantas medicinales durante un año}

Entre 1994 y 1995 (Jenaro Herrera) y entre 1996 y 1997 (Samiria y Puinahua), se trabajó con 38 familias, distribuidas en siete comunidades de las tres zonas con la finalidad de registrar la extracción/uso de productos de los bosques, así como también de conocer la preparación y el uso de las plantas medicinales. Las familias apuntaron sus actividades en un diario. Estas fueron visitadas cada dos semanas para realizarles entrevistas con formularios estandarizados que puedan cuantificar sus actividades y evaluar sus valores económicos (Gram et al., 2001; Kvist et al., 2001b). Este método (al igual que el método $\mathrm{H}$ ) registra la extracción y los usos efectuados, en lugar de registrar las percepciones sobre la supuesta utilidad de las plantas. La información recopilada sobre los usos efectuados corresponde a un periodo. Así, los investigadores solo observaron los usos/preparaciones aplicados en el momento de su visita.

\section{H. Preparados medicinales observados en una visita}

Entre 1997 y 1998, se visitaron, al azar, las casas de 102 familias (42 familias en Jenaro Herrera, 30 en Puinahua y 30 en Samiria). A cada una de ellas se visitó en una oportunidad. En todos los hogares, se registró la presencia de materiales y preparaciones de plantas medicinales; para ello, se entrevistó a las familias con la finalidad de averiguar su forma de uso y aplicación. Este método solo considera preparados y materiales de plantas medicinales observados por los investigadores.

\subsubsection{Objetivos básicos identificados}

Se identifican y ordenan 11 objetivos básicos para investigaciones de extracción, evaluación y uso de plantas medicinales. Solo se incluyen los objetivos factibles de alcanzar a partir de estudios de campo. Se ignoran los objetivos que dependen de estudios en laboratorios, clínicas u hospitales.

\section{Determinar la identidad científica}

Para los estudios científicos, es indispensable conocer la identidad científica de las plantas usadas para fines medicinales. En zonas tropicales con alta diversidad botánica, es necesario recolectar, para su posterior identificación en el herbario, muestras botánicas de una gran proporción de las plantas medicinales.

\section{Determinar la relación entre nombres vernaculares y nombres científicos}

Los informantes refieren a las plantas medicinales con sus nombres locales (vernaculares). Muchas veces brindan información sobre plantas difíciles o imposi- 
bles de encontrar y recolectar para su identificación científica. Si no se conocen los nombres locales correspondientes a las especies científicas, aquella información es poco útil para los investigadores. La relación entre los nombres locales y los nombres científicos también es imprescindible para entender el sistema de clasificación de las plantas usadas por las poblaciones locales.

\section{Evaluar la variación de conocimiento en población local}

La evaluación de la población local permite comparar el conocimiento y uso de las plantas medicinales en relación con diversas enfermedades entre grupos étnicos de la misma área, entre hombres y mujeres, entre grupos de edad, entre la población común y entre especialistas (curanderos, parteras, etc.).

\section{Evaluar la percepción y reconocimiento del uso}

A menudo, los informantes indican varias plantas medicinales como útiles contra la misma enfermedad. Sin embargo, una o algunas de estas, normalmente, son reconocidas como mejores que las demás. Muchas veces se usan estas especies preferidas, pero, en otros casos, se privilegia aquellas que se obtienen más fácilmente, aunque no estén entre las preferidas. Esto ocurre por diferentes causas, tales como escasez de las especies preferidas o las grandes distancias que hay que recorrer para obtenerlas.

\section{Determinar la frecuencia de uso y/o cantidades extraídas}

La información recogida debe referirse a las plantas que efectivamente han sido utilizadas por los informantes con la finalidad de determinar qué plantas son las más utilizadas para una enfermedad y de evaluar las cantidades extraídas y/o usadas. Esto dará una idea de la demanda y/o preferencia por determinada especie. Además, así se facilitan las estimaciones del valor económico de las plantas medicinales extraídas para consumo y/o venta.

\section{Registrar la preparación y la aplicación}

Se registra, de manera consistente y precisa, la información específica con respecto al uso, preparación y aplicación de plantas medicinales. Así, se registran el periodo de uso, la utilización independiente o conjunta (con elementos vegetales o no) de las plantas, etc. En general, el uso de una sola planta medicinal, sin que se haya mezclado con otros elementos y con una dosificación precisa, para una enfermedad bien definida indica qué efectos son más probables (Kvist et al., 1998). 


\section{Registrar el impacto de los tratamientos}

Es necesario interrogar a los curanderos y/o pacientes tratados con plantas medicinales. Se recopilan sus experiencias y percepciones con respecto a la eficiencia y efectos de los tratamientos. Dicha información puede sugerir cualidades y propiedades de algunas plantas usadas para una enfermedad, así como también que otras de ellas, aparentemente, no tienen valor curativo.

\section{Identificar las plantas que aparentemente tienen sustancias activas}

El punto de partida de los estudios farmacológicos de las plantas medicinales, que aparentemente tienen valores curativos y pueden contener sustancias activas, muchas veces son los datos etno-botánicos. Estos sugieren el impacto de los tratamientos, como por ejemplo el consenso de los informantes, dentro de una zona así como también entre diferentes zonas, con respecto a la efectividad de una especie contra una o contra varias enfermedades.

\section{Determinar de qué hábitats se realiza la extracción}

El hábitat de una especie medicinal debe ser registrado para poder establecer su ecología y su distribución, así como también para proyectar su posible manejo y/o domesticación. También puede permitir mejores identificaciones a partir de nombres vernaculares. Así, por ejemplo, la población a menudo se refiere a distintas especies como "del bajo" y "de altura", de acuerdo con su distribución en las zonas inundables y en el terreno de tierra firme, respectivamente. Si se conoce el hábitat de las plantas medicinales y se dispone de datos cuantitativos que indiquen la frecuencia de uso y las cantidades de materiales extraídos, se puede determinar qué hábitats son los más importantes para proveer plantas medicinales a la población.

\section{Registrar cómo se realiza la extracción y el manejo}

El registro de la conservación y el manejo permite rescatar los métodos tradicionales adecuados sobre este aspecto para las especies medicinales estudiadas. Por ejemplo, es posible conocer si la extracción aniquila una determinada planta medicinal o tiene poco o ningún impacto en su sobrevivencia.

\section{Comprobar si hay depredación y necesidad de proteger/manejar a la planta medicinal}

Es importante averiguar, con la finalidad de definir e implementar sistemas de aprovechamiento y conservación más adecuados, si la extracción destructiva de las plan- 
tas medicinales ha disminuido su abundancia o extinguido algunas especies en zonas aprovechadas por poblaciones locales. El consumo local amenaza a pocas especies vegetales, pero a menudo la extracción para el mercado fitoterapéutico reduce poblaciones.

\subsubsection{Parámetros para la evaluación de métodos}

Se identifican y describen nueve parámetros adicionales por considerar, cuando se eligen los métodos de un estudio. Se pueden diferenciar tres categorías: los primeros parámetros (I, II, III) están relacionados con las condiciones previas del estudio; los siguientes (IV, V) pueden ser considerados como de carácter general y los últimos (VI, VII, VIII y IX) se refieren al nivel de certidumbre de los resultados encontrados.

\section{El costo del estudio}

El punto de partida será el presupuesto disponible. Los métodos variarán con respecto a los recursos necesarios en cuanto a la mano de obra, los materiales, el transporte, etc. Se puede bajar los costos aprovechando inversiones ya realizadas, por ejemplo, a través del aprovechamiento de las parcelas permanentes existentes o de la integración a estudios con fines más amplios.

\section{Requerimiento de conocimientos y experiencias previas}

Los métodos pueden ser exigentes con respecto al dominio de la flora, a los hábitats de las plantas y a los aspectos socio-económicos en el área local. Por ejemplo, es necesario conocer de antemano la relación entre nombres vernaculares y científicos. Se puede aplicar los métodos en un orden lógico aplicando primero los métodos menos exigentes, de manera que se facilitan así los más exigentes.

\section{La facilidad para captar y procesar datos}

La selección de los métodos adecuados también depende de las experiencias y habilidades de los investigadores con respecto a la elaboración de herramientas (cuestionarios, fichas, etc.), a la interpretación de datos mediante análisis estadísticos y otros.

\section{La adecuación para registrar la alta diversidad de conocimiento}

El método puede captar una gran variación de aplicaciones de plantas medicinales o, contrariamente (parámetro $\mathrm{V}$ ), puede solo registrar las plantas y usos más conocidos y/o comunes. 


\section{La generación de resultados representativos de la población}

El método determina la importancia relativa de las plantas medicinales. Registra así la importancia de las plantas y de los usos medicinales principales de la población o, contrariamente (parámetro IV), describe la diversidad de usos.

\section{La certidumbre de las informaciones sobre los usos}

El método otorga como resultado una información confiable con respecto a la extracción y aplicación de plantas medicinales. Los métodos adecuados para describir la alta diversidad de usos (parámetro IV) pueden tener bajos niveles de certidumbre de usos, lo que ocasiona que se investiguen muchas plantas diferentes, incluso las menos conocidas.

\section{La certidumbre de las identificaciones botánicas}

El método otorga determinaciones confiables sobre la identidad científica de las plantas usadas. Hay un alto nivel de certidumbre cuando los investigadores observan, identifican y, si es necesario, recolectan muestras botánicas de las plantas medicinales. Si no existe una relación consistente entre los nombres vernaculares y los científicos, hay incertidumbre cuando los investigadores dependen de nombres vernaculares brindados por los informantes. Los métodos que brindan información representativa (parámetro V) pueden tener bajos niveles de certidumbre botánica, lo que ocasiona que los investigadores acepten información sobre plantas y/o productos medicinales no observados.

\section{La capacidad para corregir la información equivocada}

El método facilita la detección, corrección y/o desecho de la información equivocada o sin importancia real, ya sea a partir de niveles de consenso entre informantes, la búsqueda de plantas medicinales específicas para su identificación, etc.

\section{La participación activa de la población}

El método permite a la población participar en el estudio. Esta tiene libertad para expresarse, para seleccionar las plantas por estudiar, para compartir con el investigador las actividades, etc. Los informantes que son participantes activos pueden ser más dedicados y serios. Así, prevén la información más representativa y facilitan la posterior ejecución de proyectos de manejo y conservación de plantas medicinales. 


\section{RESULTADOS}

\subsection{Caracterización de los métodos evaluados}

En el Cuadro 1, se destacan las características particulares de los ocho métodos. Un método es evaluado cualitativamente y los demás cuantitativamente. La mayoría de los métodos registró usos medicinales potenciales de plantas que fueron elegidas principalmente por los informantes. Siete de los métodos recogen información del momento.

Cuadro 1: Caracterización de los ocho métodos evaluados.

\begin{tabular}{|l|c|c|c|c|c|c|c|c|}
\hline CARACTERÍSTICA / MÉTODO & $\mathrm{A}$ & $\mathrm{B}$ & $\mathrm{C}$ & $\mathrm{D}$ & $\mathrm{E}$ & $\mathrm{F}$ & $\mathrm{G}$ & $\mathrm{H}$ \\
\hline Cualitativa & $\mathrm{x}$ & & & & & & & \\
\hline & & $\mathrm{x}$ & $\mathrm{x}$ & $\mathrm{x}$ & $\mathrm{x}$ & $\mathrm{x}$ & $\mathrm{x}$ & $\mathrm{x}$ \\
\hline Cuantitativa & $\mathrm{x}$ & $\mathrm{x}$ & $\mathrm{x}$ & $\mathrm{x}$ & $\mathrm{x}$ & $\mathrm{x}$ & & \\
\hline & & & & & & & $\mathrm{x}$ & $\mathrm{x}$ \\
\hline Usos potenciales reportados & $\mathrm{x}$ & $\mathrm{x}$ & & & & & & \\
\hline & $\mathrm{x}$ & & $\mathrm{x}$ & $\mathrm{x}$ & $\mathrm{x}$ & & & \\
\hline & & & & & & $\mathrm{x}$ & $\mathrm{x}$ & $\mathrm{x}$ \\
\hline $\begin{array}{l}\text { Plantas elegidas elegidas por investigador informante } \\
\text { Plantas elegidas de otra manera }\end{array}$ & $\mathrm{x}$ & $\mathrm{x}$ & $\mathrm{x}$ & & & $\mathrm{x}$ & $\mathrm{x}$ & $\mathrm{x}$ \\
\hline & & & & $\mathrm{x}$ & $\mathrm{x}$ & & & \\
\hline $\begin{array}{l}\text { Busca información general } \\
\text { Busca información específica }\end{array}$ & $\mathrm{x}$ & $\mathrm{x}$ & $\mathrm{x}$ & & $\mathrm{x}$ & $\mathrm{x}$ & & \\
\hline $\begin{array}{l}\text { Consulta a informantes aislados } \\
\text { Consulta a nivel familiar }\end{array}$ & & & & $\mathrm{x}$ & & & $\mathrm{x}$ & $\mathrm{x}$ \\
\hline $\begin{array}{l}\text { Información del momento } \\
\text { Información del pasado }\end{array}$ & $\mathrm{x}$ & $\mathrm{x}$ & $\mathrm{x}$ & $\mathrm{x}$ & $\mathrm{x}$ & $\mathrm{x}$ & & $\mathrm{x}$ \\
\hline
\end{tabular}
A. Estudio etno-botánico cualitativo.
B. Usos potenciales de plantas medicinales elegidas por investigadores.
C. Usos potenciales de plantas medicinales elegidas por informantes.
D. Plantas útiles para enfermedades específicas.
E. Plantas medicinales conocidas bajo nombres vernaculares.
F. Usos medicinales potenciales registrados mediante inventarios participativos.
G. Recopilación de uso de plantas medicinales durante un año.
H. Plantas medicinales observadas en una visita. 


\subsection{Valoración de métodos según los objetivos identificados}

El Cuadro 2 evalúa los ocho métodos sobre la base de los 11 objetivos básicos identificados. Los métodos que contribuían de manera significativa, de manera regular o de manera exigua para cumplir los objetivos particulares están marcados con los números 1,0 y -1 , respectivamente. Además, en la parte inferior, aparece el número de objetivos para el cual el método contribuye significativa, regular o exiguamente, así como también el puntaje alcanzado. Ningún método cumple significativamente con todos los objetivos y hay una variación de un mínimo de dos objetivos hasta un máximo de seis objetivos significantes.

Cuadro 2: Valoración de métodos según su contribución a los objetivos.

\begin{tabular}{|l|c|c|c|c|c|c|c|c|}
\hline OBJETIVO / MÉTODO & A & B & C & D & E & F & G & H \\
\hline Identidad científica & 1 & 1 & 0 & 0 & 0 & 1 & -1 & -1 \\
\hline Relación N.V./ N.C. & 1 & 1 & 0 & 0 & 1 & 1 & -1 & -1 \\
\hline Variación de conocimiento & -1 & 1 & 1 & 0 & 1 & 1 & 1 & 0 \\
\hline Reconocimiento de usos & 0 & 1 & 1 & 0 & -1 & 1 & -1 & -1 \\
\hline Frecuencia de usos & -1 & -1 & 0 & 0 & -1 & -1 & 1 & 1 \\
\hline Registra preparación/aplicación & 0 & -1 & 1 & 1 & 0 & -1 & 1 & 1 \\
\hline Registra efecto/eficiencia & 0 & -1 & 1 & 1 & -1 & -1 & 1 & 0 \\
\hline Identifica sp. con sustancias activas & 0 & -1 & 0 & 1 & -1 & -1 & 1 & 0 \\
\hline Determina hábitats importantes & 0 & 0 & 1 & 0 & 0 & 0 & 1 & 0 \\
\hline Rescate extracción/manejo tradicional & 0 & 1 & 0 & 0 & 0 & 1 & -1 & -1 \\
\hline Comprueba depredación & -1 & 0 & 1 & 0 & 0 & 1 & -1 & -1 \\
\hline Número de métodos por categorías & $2,6,3$ & $5,2,4$ & $6,5,0$ & $3,8,0$ & $2,5,4$ & $6,1,4$ & $6,0,5$ & $2,4,5$ \\
\hline Puntaje & -1 & 1 & 6 & 3 & -2 & 2 & 1 & -3 \\
\hline
\end{tabular}

A. Estudio etno-botánico cualitativo.

B. Usos potenciales de plantas medicinales elegidas por investigadores.

C. Usos potenciales de plantas medicinales elegidas por informantes.

D. Plantas útiles para enfermedades específicas.

E. Plantas medicinales conocidas bajo nombres vernaculares.

F. Usos medicinales potenciales registrados mediante inventarios participativos.

G. Recopilación de uso de plantas medicinales durante un año.

H. Plantas medicinales observadas en una visita.

N.V. $=$ Nombre vulgar.

N.C. $=$ Nombre científico.

Contribución de categorías: 1 = Significativo; $0=$ Regular; $-1=$ Poco significativo . 


\subsection{Valoración de los métodos según los parámetros identificados}

El Cuadro 3 evalúa los ocho métodos a partir de los nueve parámetros identificados, marcados con los números 1,0 y -1 (métodos favorables, algo favorables y desfavorables, respectivamente). Además, se consigna, en las dos últimas filas el número de métodos por categorías de evaluación y los puntos alcanzados. Cada método representa una combinación distinta de parámetros que tienen un impacto favorable, poco favorable o desfavorable con respecto a su valor. La variación oscila desde los cinco parámetros favorables hasta los seis parámetros desfavorables.

Cuadro 3: Valoración de métodos según parámetros adicionales.

\begin{tabular}{|l|c|c|c|c|c|c|c|c|}
\hline PARÁMETRO / MÉTODO & A & B & C & D & E & F & G & H \\
\hline Bajo costo de estudio & 1 & -1 & 1 & 1 & 1 & 0 & -1 & -1 \\
\hline Condiciones previas limitadas & 1 & 1 & 0 & 0 & 0 & 0 & -1 & -1 \\
\hline Facilidad de captar/ procesar datos & 1 & -1 & 0 & 1 & 1 & -1 & 0 & 0 \\
\hline $\begin{array}{l}\text { Registra alta diversidad de } \\
\text { conocimientos }\end{array}$ & 0 & 1 & 0 & -1 & -1 & 0 & 0 & -1 \\
\hline $\begin{array}{l}\text { Genera datos representativos de } \\
\text { población }\end{array}$ & 0 & 0 & 1 & -1 & -1 & 0 & 1 & 1 \\
\hline Certidumbre de información de uso & -1 & 1 & 1 & 1 & 1 & 0 & -1 & -1 \\
\hline Certidumbre de identidad botánica & 1 & 1 & -1 & 0 & 1 & 0 & -1 & -1 \\
\hline Capacidad de corregir información & -1 & 1 & 1 & 1 & 1 & 0 & -1 & -1 \\
\hline Participación activa de informantes & 0 & -1 & 1 & 0 & 0 & 1 & 1 & -1 \\
\hline \multicolumn{1}{|c|}{ Número de métodos por categorías } & $4,3,2$ & $4,1,4$ & $5,3,1$ & $2,4,3$ & $5,2,2$ & $1,6,2$ & $3,2,4$ & $2,1,6$ \\
\hline Puntaje & 2 & 0 & 4 & -1 & 3 & -1 & -1 & -4 \\
\hline
\end{tabular}
A. Estudio etno-botánico cualitativo.
B. Usos potenciales de plantas medicinales elegidas por investigadores.
C. Usos potenciales de plantas medicinales elegidas por informantes.
D. Plantas útiles para enfermedades específicas.
E. Plantas medicinales conocidas bajo nombres vernaculares.
F. Usos medicinales potenciales registrados mediante inventarios participativos.
G. Recopilación de uso de plantas medicinales durante un año.
H. Plantas medicinales observadas en una visita.

Impacto de parámetros adicionales: $1=$ Favorable; $0=$ Poco favorable; $-1=$ Desfavorable. 


\section{DISCUSIÓN}

En el Cuadro 2, se observa que el método C posee la mayoría de puntos positivos y el método $\mathrm{H}$ la mayor cantidad de puntos negativos. Se representa así, respectivamente, tanto la mayor contribución potencial como la más limitada respecto de los 11 objetivos definidos. En el Cuadro 3, los métodos $\mathrm{C}$ y $\mathrm{H}$ destacan nuevamente; sus puntos suman la mayor cantidad de positivos y la mayor cantidad de negativos, respectivamente. Ello indica que estos dos métodos son los que tienen más ventajas y desventajas. El hecho de que el método $\mathrm{C}$ contribuya a todos los 11 objetivos definidos, aunque solo de manera significativa a 6 de ellos y, además, solo sea desfavorecido según un parámetro, indica que será el mejor método si pretendemos contribuir a todos los objetivos. Sin embargo, pocos estudios priorizan los 11 objetivos y, en función de la combinación de los objetivos vigentes, otro método puede ser el más favorable para aplicar. Además, casi siempre una combinación de métodos es mejor para cumplir con todos los objetivos vigentes.

Para facilitar esa selección, destacamos las ventajas y desventajas principales de cada uno de los ocho métodos.

\section{A. Estudio etno-botánico cualitativo}

Tanto los gastos del estudio como la demanda de conocimientos previos son limitados. Cabe destacar que no existe la necesidad de procesar mucha información. Así, sin conocer previamente la flora y la población del estudio, se podría encontrar y recolectar muchas plantas medicinales diferentes, de las que además se puede conseguir sus nombres vernaculares. Por eso es posible realizar un estudio cualitativo antes de aplicar los métodos más exigentes y complejos.

\section{B. Usos potenciales de plantas medicinales elegidas por los investigadores}

Este método es exigente con respecto al procesamiento de los resultados e implica altos gastos si el proyecto tuviera que establecer parcelas permanentes. Sin embargo, es óptimo para establecer la relación entre los nombres vernaculares y las identidades científicas de las plantas medicinales, pues los investigadores conocen la identidad científica de los individuos de árboles y sogas que se discuten con los informantes (Kvist et al., 1995; 2001a). Además, el nivel de consenso entre los informantes permite determinar qué nombres vernaculares son bien conocidos y se puede comparar el conocimiento, por ejemplo, entre los hombres y las mujeres (Stagegaard et al., 2001). 
Una de las limitaciones de este método es el hecho de que no todas las especies útiles están representadas en las parcelas (baja densidad y distribución en hábitats sin parcelas). Otra desventaja consiste en que los informantes, como consecuencia del hecho de ser entrevistados sobre usos de árboles y bejucos elegidos por los investigadores, que incluso en muchos casos les son poco conocidos, reportan muchos usos que raras veces o nunca son efectuados. Esto otorga como resultado un bajo nivel de consenso. Por ejemplo, de las 170 especies indicadas como medicinales, solo 10 fueron reportadas por seis informantes (Kvist et al., 1995). Además, en las comunidades ubicadas cerca de las parcelas, solo 30 de estas 170 especies fueron mencionadas (método C), (Kvist et al., 1998).

\section{Usos potenciales de plantas medicinales elegidas por los informantes}

Como ya se mencionó, este método contribuye a la consecución de muchos objetivos y posee pocas desventajas. La información recogida tiene un alto nivel de certidumbre en vista de que los mismos informantes seleccionan las plantas medicinales por discutir y participan durante un periodo tan limitado que el tiempo solo alcanza para reportar las plantas que conocen bien, sean estas especies cultivadas o silvestres (Kvist et al., 1998). Además, el nivel de consenso sirve para identificar a las especies que se menciona a través de la comparación del conocimiento de los distintos grupos de informantes. Por ejemplo, puede compararse el testimonio de nativos y mestizos. Asimismo, es posible obtener información detallada sobre la preparación, aplicación y los efectos de las plantas. La desventaja principal es la necesidad de contar con una relación confiable entre nombres vulgares y científicos, pues no todas las plantas se llegan a observar. Además, se recibe poca información con respecto a los usos menos conocidos o practicados de las plantas medicinales.

\section{Plantas útiles contra enfermedades específicas}

Este método brinda información específica de las plantas usadas contra determinadas enfermedades seleccionadas (en el presente caso: malaria y leishmaniasis). Se entrevistan informantes de varias comunidades sobre sus conocimientos y prácticas. Por esta característica, el método contribuye a muchos objetivos. Su ventaja principal es la captación de datos detallados tanto sobre la preparación y aplicación como sobre los supuestos efectos de las plantas medicinales. Sobre la base de esta información, se puede recoger el nivel de consenso y seleccionar especies promisorias para estudios fitoquímicos. Las investigaciones de malaria y leishmaniasis (Gonzales et $a l ., 1999,2000)$ han demostrado que estudios específicos son necesarios para investigar las plantas usadas contra enfermedades relativamente raras (los estudios generales ofrecen pocos datos con respecto a la malaria y ninguna información con res- 
pecto a leishmaniasis). Además, se puede identificar las especies más conocidas por niveles de consenso.

\section{E. Plantas medicinales conocidas bajo nombres vernaculares}

Este método, por su carácter específico respecto de las plantas seleccionadas, también contribuye a muchos objetivos. Es valioso para descubrir confusiones entre especies diferentes que tienen un mismo nombre vernacular. Por ejemplo, el «mururé» es una especie de Brosimum en Jenaro Herrera, pero una especie de Cynometra en Samiria; «cumaceba del bajo» es Lecointea amazónica en Jenaro Herrera, pero es una especie de Swartzia en Samiria y Puinahua. De esta manera, se corrige y mejora la relación entre los nombres científicos y los vernaculares, de manera que se evita confundir los efectos curativos atribuidos a varias y diferentes plantas. Además, se puede describir la variación del conocimiento de las especies entre zonas geográficas o grupos de informantes distintos. La desventaja principal es el alto costo que implica, debido a que debe visitarse zonas de diferentes características geográficas que, por lo general, están bastante alejadas.

\section{F. Usos medicinales potenciales registrados mediante inventarios participativos}

Este método tiene la capacidad de contribuir a la consecución de varios objetivos. Puede aplicarse de diferentes formas. Por ejemplo, por medio de inventarios rápidos que permitan aceptar los nombres vulgares brindados por los informantes o, por el contrario, identificar a cada individuo o especie científica, de manera que se avance con más cautela principalmente en bosques con alta diversidad. En el primer caso, se puede inventariar el estado de las poblaciones de plantas medicinales; en el segundo caso, se puede investigar la relación entre los nombres vernaculares y los nombres científicos. Una limitación que siempre existe consiste en que el área de estudio es restringida por el investigador y no se recoge la información relacionada con las plantas distribuidas en otras áreas.

\section{G. Recopilación de uso de las plantas medicinales durante un año}

Este método ofrece una mejor evaluación tanto de la frecuencia de uso de las diferentes plantas medicinales como de las cantidades extraídas, lo que facilita estimaciones económicas del valor de la extracción y uso (o venta) de las especies estudiadas. También la colaboración continua con los mismos informantes facilita datos precisos y detallados sobre la preparación, aplicación y efectos de las plantas medicinales, además de destacar la importancia de los hábitats de donde se extraen. El método es costoso y requiere de mucho tiempo. Además, la desventaja principal es la incertidumbre con respecto a la identidad científica, pues los investigadores, pocas veces, 
observan las plantas y hay una tendencia a solo recibir información sobre plantas medicinales bien conocidas.

\section{H. Preparados medicinales observados en una visita}

Este método contribuye a lograr una cantidad reducida de objetivos. Tiene más desventajas que todos los demás. Sin embargo, puede facilitar el recojo de información precisa y detallada con respecto a la preparación y aplicación de las plantas medicinales vía observaciones in situ. También puede determinar la frecuencia de usos de varias plantas medicinales, aunque las condiciones específicas en el momento del estudio se pueden distorsionar, por ejemplo, debido a las epidemias o a la época del año en que se visita a los informantes.

\section{CONCLUSIONES Y RECOMENDACIONES}

- La elección/elaboración de los métodos a seguir debe realizarse a partir de un estudio.

- Para definir los objetivos en un estudio de plantas medicinales es recomendable tomar en cuenta dos consideraciones importantes: 1. Si se busca información representativa (muchos informantes) o precisa y detallada (pocos informantes). 2. Si se prioriza la determinación de la identidad científica o la cuantificación de la importancia de las diferentes plantas (que siempre implica aceptar algunos usos a partir de sus nombres vernaculares).

- Las combinaciones de métodos son posibles, y a menudo preferibles, puesto que un solo método no es capaz de cubrir toda la información deseada. La combinación ideal de métodos dependerá de los objetivos, del conocimiento de la flora y la cultura locales, así como también de los recursos disponibles.

- Es una buena estrategia, principalmente para establecer la relación entre los nombres científicos y los vernaculares, realizar un estudio cualitativo antes de iniciar estudios cuantitativos.

- Cuando no existe un conocimiento previo de la cultura de la comunidad ni de la botánica de la zona, es recomendable aplicar entrevistas piloto que permitan corregir errores y definir herramientas simples y concretas para un óptimo registro de la información. 
- Es recomendable incorporar un estudio etnobotánico dentro de estudios más amplios, pues esto abarata costos, permite interactuar con pobladores locales e instituciones afines, además de que tiene un efecto multiplicador de experiencias.

- Se necesita buscar y recolectar las plantas conocidas por sus nombres vernaculares en varios lugares de la selva para determinar si estas pertenecen a una misma especie o a especies diferentes.

- La mayoría de los métodos discutidos solo fue comprobada en zonas inundables. Sin embargo, por sus características, los métodos podrían ser válidos en otras condiciones culturales y ecológicas.

\section{BIBLIOGRAFÍA}

ALCORN, J.B. 1981. Some factors influencing botanical resources perception among the Haustec. En: Journal of Ethnobiology 1: 221-230.

BERNARD, H.T.; KILLWORTH, P.; KRONENFELD, D.; SAILER, L. 1984. The problem of informant accuracy: The validity of retrospective data. En: Annual Review of Anthropology 13: 495-517.

GODOY, R.; LUBOWSKI, R. 1992. Guidelines for the economic valuation of nontimber forest products. En: Current Anthropology 33: 423-433.

GONZALES, A.; KVIST, L.P.; ORÉ, I.; DELGADO, O.; MEJÍA, K. 1999. Plantas medicinales, utilizadas en el tratamiento de leishmaniasis en el departamento de Loreto (Perú). En: Conocimiento 5: 209-220.

GONZALES, A.; KVIST, L.P.; FLORES, M., ORÉ, I.; DELGADO, O. 2000. Usos de plantas medicinales para el tratamiento de malaria, en dos distritos de la provincia de Maynas. Loreto (Perú). En: Conocimiento 6: 109-124.

GRAM, S. 2001. Economic Valuation of Forest Products. Assessment of Methodological Shortcomings. En: Ecological Economics 36: 109-117.

GRAM, S.; KVIST, L.P.; CÁCERES, A. 2001. The economic importance of products extracted from Amazonian flood plain forests. En: Ambio V. 6 (en prensa). 
JOHNS, T.; KOKWARO, J.; KIMANANI, E. 1990. Herbal remedies of the Luo of Siaya District, Kenya: Establishing quantitative criteria for consensus. En: Economic Botany 44: 369-381.

KVIST, L.P. 1997. A comparison of qualitative and three quantitative ethnomedicinal methodologies based on studies in Peru and Ecuador. En: Ríos, M. y Pedersen, H.B. (ed.). Uso y Manejo de Recursos Vegetales. Memorias del Segundo Simposio Ecuatoriano de Etnobotánica y Botánica Económica. Quito (Ecuador): Ediciones Abya-Yala. pp. 361-382.

KVIST, L.P.; NEBEL, G. 2000. Bosques de la llanura aluvial del Perú: Ecosistemas, habitantes y uso de recursos. En: Folia Amazónica 10: 5-56.

KVIST, L.P.; ANDERSEN, M.; HESSELSOE, M.; VANCLAY, J.K. 1995. Estimating use-values and relative importance of Amazonian flood plain trees and forests to local inhabitants. En: Commonwealth Forestry Review 74: 293-200.

KVIST, L.P.; ORÉ, I.; LLAPAPASCA, C. 1998. Plantas utilizadas en transtornos ginecológicos, parto y control de natalidad en mujeres de la parte baja del Río Ucayali - Amazonía Peruana. En: Folia Amazónica 9: 115-142.

KVIST, L.P.; ANDERSEN, M.; STAGEGAARD, J.; LLAPAPASCA, C. 2001a. Extraction from woody forest plants in flood plain communities in Amazonian Peru: use, choice, evaluation and conservation status of resources. En: Forest Ecology and Management 5465: 1-28 (en prensa).

KVIST, L.P.; GRAM, S.; ORÉ, I.; CÁCERES, A. 2001b. Socio-economy of flood plain households in the Peruvian Amazon. En: Forest Ecology and Management 5466: 1-12 (en prensa).

NEBEL, G.; KVIST, L.P.; VANCLAY, J.K.; CHRISTENSEN, H.; FREITAS, L.; RUIZ, J. 2000 a. Structure and floristic composition of flood plain forests in the Peruvian Amazon: I. Overstorey. Forest Ecology and Management. En: Folia Amazónica 10: 91-150.

NEBEL, G.; DRAGSTED, J.; VANCLAY, J. 2000 b. Estructura y composición florística del bosque de la llanura aluvial inundable de la Amazonía Peruana: II. El sotobosque de la restinga. En: Folia Amazónica 10: 151-182. 
ORÉ, I.; DELGADO, O.; LLAPAPASCA, C.; KVIST, L.P.; GONZALES, A. 1999. Composición, similaridad y uso de especies cultivadas en huertas domésticas de la Reserva Nacional Pacaya-Samiria. En: Conocimiento 5: 141-157. Iquitos (Perú): Universidad Nacional de la Amazonía Peruana.

PHILLIPS, O.; GENTRY, A. 1993a. The useful plants of Tambopata, Peru: I. Statistical hypothesis tests with a new quantitative technique. En: Economic Botany 47: 15-32.

PHILliPS, O.; GENTRY, A. 1993b. The useful plants of Tambopata, Peru: II. Additional hypothesis testing in quantitative ethnobotany. En: Economic Botany 47: 33-43.

STAGEGAARD, J.; KVIST, L.P.; SOERENSEN, M. 2001. Estimations of the importance of plant resources extracted by inhabitants of Peruvian Amazon flood plains. En: Economic Botany. 


\section{ESTUDIO CUANTITATIVO DE LA MACROFAUNA DEL SUELO EN DIFERENTES SISTEMAS DE USO DE LA TIERRA EN LA AMAZONÍA PERUANA}

\section{Beto Pashanasi*}

\section{RESUMEN}

La comunidad de macro-invertebrados del suelo fue evaluada en 22 sistemas de uso del suelo en las zonas de Yurimaguas y Pucallpa. Se separaron, manualmente, 10 muestras por sistema de uso de $25 \mathrm{~cm}$ x $25 \mathrm{~cm}$ x $30 \mathrm{~cm}$ durante la estación lluviosa.

El bosque primario, no intervenido e intervenido, tiene una diversidad muy rica. Asimismo, su densidad (382 a 853 individuos $/ \mathrm{m}^{2}$ ) y su biomasa, dominada por oligochaetas, isópteras y miriápodos $\left(57,8\right.$ a $91,1 \mathrm{~g}$ peso fresco $\left./ \mathrm{m}^{2}\right)$, son bastante altas. Los cultivos de esta comunidad, cuya densidad es de 362 a 574 individuos $/ \mathrm{m}^{2}$ y cuya biomasa es de 5,1 a 32,4 g peso fresco $/ \mathrm{m}^{2}$, se encuentran severamente agotados.

Las pasturas tienen baja diversidad. La densidad de su población varía en un rango de 654 a 1034 individuos $/ \mathrm{m}^{2}$. Su biomasa es tan alta como de 38,4 a 165,9 g peso fresco $/ \mathrm{m}^{2}$, debido a la colonización de la lombriz peregrina, Pontoscolex corethrurus. En las purmas, la densidad poblacional está en un rango de 334 a 838 individuos $/ \mathrm{m}^{2}$; mientras que la biomasa varía entra 4,2 y $102 \mathrm{~g}$ peso fresco $/ \mathrm{m}^{2}$. Cabe destacar que, en algunos casos, la riqueza taxonómica es mayor que la del bosque primario.

Finalmente, los sistemas agroforestales con cobertura de leguminosas tienen la más alta diversidad. Lo contrario ocurre en los sistemas con cobertura de malezas que están por debajo del bosque secundario. Su densidad poblacional se encuentra en un rango que va desde 557 hasta 2896 individuos $/ \mathrm{m}^{2}$, mientras que su biomasa varía entre 18,5 y $170,5 \mathrm{~g}$ peso fresco $/ \mathrm{m}^{2}$, debido a la conservación de gran parte de la fauna del bosque primario que, además, es colonizada por especies oportunistas de terrenos disturbados (miriápodos, oligochaetas e isópteras).

Palabras clave: Macrofauna, suelo, sistemas, bosques, purmas.

* Universidad Nacional de la Amazonía Peruana. Facultad de Zootecnia.Yurimaguas, Loreto (Perú). Correo electrónico: bpashanasi@terra.com.pe 


\begin{abstract}
The community of soils macroinvertebrates was evaluated in 22 different systems of use of the soil, in the zone of Yurimaguas and Pucallpa; by manual separation of 10 samples by system of use of $25 \mathrm{~cm}$ x $25 \mathrm{~cm} \times 30 \mathrm{~cm}$, during the rainy season.

The primary forest not intervened and intervened has great diversity, and a density (382 to 853 individual $/ \mathrm{m}^{2}$ ) and a high biomass $\left(57,8\right.$ to $\left.91,1 \mathrm{~g} / \mathrm{m}^{2}\right)$ dominated by worms, isopteras and myriapods. In cultivations this community is severely exhausted, with a density from 362 to 574 individual $/ \mathrm{m}^{2}$ and a biomass from 5,1 to $32,4 \mathrm{~g} / \mathrm{m}^{2}$.

The pastures have lowers diversity, with density of population in a rank from 654 to 1034 individual $/ \mathrm{m}^{2}$ and a biomass as high as from 38,4 to $165,9 \mathrm{~g} / \mathrm{m}^{2}$, due to the colonization of the «Peregrine worm» Pontoscolex corethrurus. In the fallows purmas the population density is in a rank from 334 to 838 individual $/ \mathrm{m}^{2}$; and a biomass from 4,2 to $102 \mathrm{~g} / \mathrm{m}^{2}$; being the wealth taxonomy greater that of the primary forest in some cases.

Finally the agroforestry systems, with cover of leguminous have the highest diversity, being the contrary thing in the systems with cover of weeds that are under the secondary forest. The population density is found in a rank from 557 to 2896 individual $/ \mathrm{m}^{2}$ and a biomass from 18,5 to $170,5 \mathrm{~g} / \mathrm{m}^{2}$, due to the conservation of great part of the fauna of the primary forest and besides they are colonized for opportunist's species of disturbed lands (myriapodos, worms and isopteras).
\end{abstract}

Key words: Macrofauna, soil, systems, forests, purmas.

\title{
1. INTRODUCCIÓN
}

En los ambientes naturales del trópico húmedo, los macro-invertebrados del suelo son los mejores agentes reguladores de los procesos físico-químicos que afectan la fertilidad de los suelos (Lavelle, 1984; Lee, 1985). Ellos, por acción de la ingestión y deyección del suelo, contribuyen a la conformación de estructuras macro-agregadas resistentes. Los macro-invertebrados mezclan los residuos orgánicos, producto de la ingestión y la deyección, al excavar madrigueras para transportar suelo a la superficie por medio de cámaras subterráneas. 
En los bosques, la diversidad y la abundancia de las comunidades de macro-invertebrados puede ser usada como indicadora de la calidad del suelo (Stork y Eggleton, 1992), toda vez que las lombrices de tierra y la fauna del suelo, en general, influyen en la dinámica de sus procesos químicos. Sin embargo, la naturaleza y los mecanismos de las interacciones entre los microorganismos del suelo y la dinámica de los procesos químicos en los suelos de la Amazonía son aún poco conocidos y, posiblemente, dependientes de las cantidades y calidades de la hojarasca depositada sobre el suelo (Volhland y Schroth, 1999). Según Swift et al., (1979), la tasa de descomposición de los residuos vegetales está influenciada por la calidad del recurso, por los organismos descompositores presentes y por las condiciones ambientales.

En la Amazonía central de Brasil, los «oribatideos» (Oliveira y Franklin, 1993) y los «colémbolos» (Oliveira, 1983) tienen la mayor densidad de la fauna en la hojarasca y en el suelo de áreas no inundables. Las «isópteras» son consideradas importantes descompositoras de la hojarasca (Luizão, 1995) y, en forma conjunta con los «formicides», constituyen los grupos de mayor densidad de la fauna del suelo en la Amazonía central de Brasil (Fittkau y Klinge, 1973; Bandeira y Harada, 1991). Las «oligochaetas» tienen una abundante biomasa en la Amazonía Peruana (Lavelle y Pashanasi, 1989) y en la Amazonía central de Brasil (Barros, 1999). En los varillales de la Amazonía central de Brasil, Luizão (1995) encontró que los «diplópodos»e «isópodos» constituyen una alta densidad y una alta biomasa. De otro lado, en los sistemas agroforestales y barbechos, los «isópodos» presentaron las mayores densidades y biomasas en la macrofauna de la hojarasca, seguidos por los «diplópodos» y por las «isópteras» (Tapia-Coral et al., 1999). Estos organismos, habitantes de la hojarasca y de las capas superiores del suelo, son -debido principalmente a sus interacciones con la microflora-, de importancia crucial para las condiciones de crecimiento de las especies cultivadas y el desenvolvimiento y funcionamiento de los agro-ecosistemas, ya que cumplen un papel vital en la descomposición de la hojarasca y en la liberación de los nutrientes (Swift et al., 1979; Lavelle, 1984; Tian et al., 1997, 1998). Así, una abundante y activa fauna de la hojarasca y del suelo puede ayudar a asegurar un reciclaje rápido de los nutrientes de las plantas (Fittkau y Klinge, 1973), lo que es particularmente importante para áreas cultivadas cuyos insumos son bajos y cuyos suelos son infértiles (Volhland y Schroth, 1999). La capa superficial de hojarasca también confiere protección física al suelo contra la erosión y ayuda en el mantenimiento de su humedad (Ross et al., 1992). De esta forma, dicha capa también está contribuyendo al mantenimiento de la actividad de los organismos del suelo. Por otro lado, estudios recientes demuestran la importancia de la biota del suelo en la recuperación de las áreas degradadas (Barros, 1999; Tapia-Coral et al., 1999; Barros et al., 2000; Araujo-Vergara, 2000; Castilho, 2000). 